\title{
Perspectives from Bologna on the future directions of aortic surgery
}

The $10^{\text {th }}$ Postgraduate Course on 'Surgery of the Thoracic Aorta' in Bologna in 2019 brought together renowned international leaders in the field of aortovascular and cardiac surgery, facilitating the exchange of expert opinions and the continuation of stimulating debate over some of modern surgery's most high-stakes and challenging procedures. The Series published following the Course comprises a wide range of topics, representing the areas of aortovascular surgery subject to the most debate, such as congenital aortic surgery, arch and thoracoabdominal dissection repair, and endovascular versus open abdominal aortic repair.

In their comprehensive and detailed review of the management of adults with aortic coarctation, Velayudhan et al. outlined and evaluated the gold-standard approaches for surgical repair of what continues to be a formidable entity to manage effectively (1). Coarctation of the aorta accounts for $5-8 \%$ of all congenital heart defects and is associated with a mean life expectance of 35 years if left untreated (2). Currently, open surgical and endovascular repair are the most commonly-taken routes to repair. Open resection of the lesion with subsequent end-to-end anastomosis, introduced in 1945 by Crafoord and Nylin, is a viable approach for paediatric cases however is a suboptimal strategy for repair of aortic coarctation in adults as a result of reduced aortic elasticity and extensive collateralisation (3). The subclavian flap repair technique, though more promising in older children, is similarly not as effective in adults owing to the high risk of claudication of the arm in the long term. Prosthetic interposition tube grafting, therefore, is the preferred approach for the repair of aortic coarctation in adults (1). The use of a vascular graft to bypass the lesion with end-to-end or side-to-side anastomosis is favoured in adults as it relies on aortic dimensions remaining more or less constant following implantation. therefore, it is unsuitable for children due to the risk of outgrowth. Velayudhan et al. also outline a hybrid approach to repair of aortic coarctation: percutaneous transaortic stenting, combined with open repair of intracardiac pathologies if needed, is reported to be associated with reduced trauma and relatively rapid recovery (1). It is worth highlighting that without national or international guidelines, patient-specific treatment plans are required-Velayudhan et al. emphasise a need for prospective randomised studies to identify the optimal strategy for the surgical management of adults with aortic coarctation.

Smith and Heijmen et al. favour a staged, hybrid approach to the repair of DeBakey type I aortic dissection (4). The classical technique of entry tear resection, aortic wall re-approximation, and aortic valve resuspension represents a viable approach to a complex pathology, and can be achieved with hemi-arch repair in the majority of cases. However, it is emphasised that this technique fails to address the downstream aortic dissection often seen in patients with DeBakey type I dissections, meaning late distal sequelae (e.g., disease progression) and the need for reintervention remain omnipresent risks (4). Whether a more comprehensive technique involving distal repair during the index procedure-essentially total arch repair with frozen elephant trunk (FET) - truly offers an improved cost-benefit profile remains unclear, most especially when one recalls that a large proportion of DeBakey type I dissections present acutely out-of-ours and are therefore often operated on by non-aortovascular specialists. It is unsurprising therefore that this formidably complex procedure to address a notoriously fatal disease is associated with has been associated with up to $12 \% 30$-day mortality, $9 \%$ permanent neurological deficit rate, and an overall $13 \%$ rate of reintervention (5). Smith and Heijmen et al. hence consider the FET technique too complex, high-risk, and expensive to be applied to all acute DeBakey type I patients. Alternatively, a hybrid approach involving two-thirds arch repair with a liberal Zone 2 landing zone [thereby facilitating completion with elective thoracic endovascular aortic repair (TEVAR)] may be more feasible for non-aortovascular specialists in the acute setting (6). Such an option may be associated with improved neurological outcomes compared to full, acute FET, paving the way for a more efficacious management of acute DeBakey type I dissection.

The time-to-treatment element of managing acute Stanford type A aortic dissection (ATAAD) is no stranger to even the most novice surgeon, emergency physician, or paramedic. The propensity for ATAAD to mimic other more common and similarly serious syndromes (e.g., acute coronary syndromes) at initial presentation makes the timely identification, diagnosis, and treatment of ATAAD notoriously challenging. De Vos et al. conducted a single-centre retrospective analysis of 52 patients 
with ATAAD over the course of 10 years to identify the sources of delays between onset-to-presentation, presentation-todiagnosis, diagnosis-to-surgery, and onset-to-knife intervals, and evaluated the possible effect of these delays on clinical outcomes (7). The interval between diagnosis to surgery was found to be the most significant source of delay $(\mathrm{P}<0.05)$, while patient gender imaging was noted to exert considerable influence over onset-to-knife time. De Vos et al. note that though computed tomography (CT) is the gold-standard imaging modality for the diagnosis of ATAAD, it considerably delays the interval between presentation and surgery (7). Less time-consuming modalities such as transthoracic echocardiography or, better yet, transoesophageal echocardiography can accurately diagnose aortic dissection, and also be conducted with the patient in theatre (8). It is important to also account for structural factors often out of the hands of the attending care team; De Vos et al. report a 3.5-hour delay between diagnosis and surgery, while Harris et al. a corresponding interval of 4.3 hours $(7,9)$. Improvements in staffing management and organisation, and operating theatre availability, though difficult to implement acrossthe-board, would undoubtedly improve the onset-to-treatment delay associated with this progressively lethal, time-sensitive entity.

Morshuis' review on the place of classical elephant trunk (cET) for aortic arch repair challenges the widely-held belief that FET represents modern surgery's best effort for open surgical total arch repair (10). Though the FET technique is an effective single-stage procedure for the definitive repair of aortic arch aneurysms or dissections, and most notably eliminates mortalities associated with the delay between cET and secondary endovascular stenting (estimated to be 3.2\%), Morshuis argues that FET was associated with net higher rates of complications such as paraplegia and early mortality $(10,11)$. Even with shortened stent-graft lengths, thought to decrease the risk of spinal ischaemia, recurrent laryngeal nerve palsy and stroke rates remained high (as high as 17\% in degenerative aneurysm cases) (12). Several unanswered questions are highlightedsuch as the effect of FET stent-graft stiffness on cardiac function, and reintervention risks, which remain high. It is key, therefore to balance the short-term risks against long-term benefits, and on this front Morshuis remains an advocate of the cET technique, which persists and remains far from obsolete. But do modern innovative alternatives truly provide an improved cost-benefit profile? Undoubtedly, debate on whether FET is truly superior for cET will continue, and for now perhaps a case-by-case, patient-specific approach is optimal.

Kalra et al. evaluated and analysed the TEVAR-first approach to ATAAD complicated with mesenteric malperfusion in their narrative review (13). ATAAD complicated by malperfusion syndrome is associated with prolonged intervals from onset to diagnosis, and from diagnosis to surgery. Traditionally, this phenomenon has been managed by urgent repair of the proximal aortic tear prior to exploratory laparotomy with bowel resection, depending on the extent of mesenteric ischaemia. Unsurprisingly, this approach is associated with myriad intraoperative risks, and involves two majorly invasive phases which undoubtedly increases mortality rates among higher-risk groups. Persistent false lumen patency, re-entry tears, and branch vessel involvement are likely, and play a significant role in intraoperative or early mortality. The Emory protocol, promulgated in 2019 and outlined by Kalra et al. as a more viable route to successfully manage ATAAD with malperfusion syndrome, utilises initial TEVAR to normalise perfusion in the thoracoabdominal aorta, before open proximal aortic repair in haemodynamically stable patients (13). Early reperfusion of visceral tissue is thought to mitigate the sequelae associated with profuse mesenteric ischaemia (e.g., persistent acidosis, bowel necrosis, and oxidative stress). Aggressive fluid resuscitation to maintain normotension and reverse acidosis is then carried out, before proximal aortic repair under cardiopulmonary bypass and hypothermic circulatory arrest (HCA) (13). Notably, such an interval would also allow for complex proximal aortic repair to take place outside of the out-of-hours setting, in the hands of experienced aortovascular specialists. In cases of haemodynamic instability (e.g., rupture), antegrade deployment of TEVAR is performed during the HCA interval. In either case, it should be kept in view that the risk of mesenteric ischaemia is substantial even normotension and acidosis are correct preoperatively. All things considered, the Emory protocol for TEVAR-first approach to ATAAD with mesenteric malperfusion syndrome is promising, and results on its long-term effectiveness are eagerly awaited.

Kothari et al. offered an insightful comparison between traditional open surgical repair of thoracoabdominal aortic aneurysm (TAAA) against emerging endovascular techniques, which offer less-invasive alternative options $(14,15)$. It is unsurprising that the extreme invasiveness, high risks of mortality, and debilitating neurological and renal complications 
has driven the search for less-invasive, endovascular alternatives for the repair of extensive TAAA. Yet, the durability and long-term stability of open repair, coupled with recent advancements in strategies to mitigate its associated surgical risks, mean that open repair of TAAA is likely here to stay for some time. Indeed, endovascular repair of TAAA with extensive branch involvement is challenging, even with the use of fenestrated or branched stent grafts. Interestingly, FDA approval for endovascular repair of TAAA has yet to materialise. Kothari et al. highlight that intraoperative mortality for the open repair of TAAA stands at $7.5 \%$, permanent paraplegia at $2.9 \%$, and renal failure at $5.7 \%$ (14). Emergent repair is thought to double the risk of intraoperative mortality. Yet, open repair has been associated with a freedom from reintervention rate of $94 \%$ over 15 years. This is contrasted by a $76.8 \%$ rate of freedom from reintervention (five years) associated with branched endovascular repair, which is also thought to carry a $4.8 \%$ early mortality rate and little real difference in long-term survival or freedom from complications (16). Aneurysmal expansion, infection, and fistula formation have been identified as the key reasons for endovascular-to-open reintervention. Perhaps, then, it may be argued that though endovascular repair of TAAA is presently less durable than that which can be achieved by open repair, it represents a viable option for lower-risk TAAA repair, or as an efficacious option for reintervention following open repair in certain groups. Yet, Kothari et al. emphasise that endovascular intervention for TAAA may better suit octogenarians or other groups deemed unfit for such an extensively invasive procedure.

Undoubtedly, the $10^{\text {th }}$ Postgraduate Course on 'Surgery of the Thoracic Aorta' in Bologna has yielded intriguing results and has laid important groundwork for exciting future developments for the repair of congenital aortic defects, acute and chronic aortic dissection, and thoracoabdominal aortic repair. Though much further research is needed, we can be confident in the fact that the foundations laid in Bologna will serve as solid foundations for the advancement of personalised aortic care.

\section{Acknowledgments}

Funding: None.

\section{Footnote}

Provenance and Peer Review: This article was commissioned by the editorial office, Fournal of Visualized Surgery for the series "The 10th Postgraduate Course on 'Surgery of the Thoracic Aorta' in Bologna”. The article did not undergo external peer review.

Conflicts of Interest: All authors have completed the ICMJE uniform disclosure form (available at https://jovs.amegroups.com/ article/view/10.21037/jovs-21-62/coif). The series "The 10th Postgraduate Course on 'Surgery of the Thoracic Aorta' in Bologna" was commissioned by the editorial office without any funding or sponsorship. MB, DP and RB served as the unpaid Guest Editors of the series. The authors have no other conflicts of interest to declare.

Ethical Statement: The authors are accountable for all aspects of the work in ensuring that questions related to the accuracy or integrity of any part of the work are appropriately investigated and resolved.

Open Access Statement: This is an Open Access article distributed in accordance with the Creative Commons AttributionNonCommercial-NoDerivs 4.0 International License (CC BY-NC-ND 4.0), which permits the non-commercial replication and distribution of the article with the strict proviso that no changes or edits are made and the original work is properly cited (including links to both the formal publication through the relevant DOI and the license). See: https://creativecommons.org/ licenses/by-nc-nd/4.0/.

\section{References}

1. Velayudhan BV, Idhrees AM. Coarctation of aorta in adults: a narrative review of surgical and endovascular management. J Vis 
Surg 2021;7:17.

2. Baumgartner H, Bonhoeffer P, De Groot NM, et al. ESC Guidelines for the management of grown-up congenital heart disease (new version 2010). Eur Heart J 2010;31:2915-57.

3. Crafoord C, Nylin G. Congenital coarctation of the aorta and its surgical treatment. J Thorac Surg 1945;14:347-61.

4. Smith T, Heijmen RH, Smith T, et al. Staged, hybrid approach to acute DeBakey Type I aortic dissection. J Vis Surg 2021;7:22.

5. Parikh N, Trimarchi S, Gleason TG, et al. Changes in operative strategy for patients enrolled in the International Registry of Acute Aortic Dissection interventional cohort program. J Thorac Cardiovasc Surg 2017;153:S74-9.

6. Desai ND, Hoedt A, Wang G, et al. Simplifying aortic arch surgery: open zone 2 arch with single branched thoracic endovascular aortic repair completion. Ann Cardiothorac Surg 2018;7:351-6.

7. De Vos M, Ranschaert W, Vergauwen W, et al. Acute type A aortic dissection: timeline between onset and treatment. J Vis Surg 2021;7:24.

8. Froehlich W, Tolenaar JL, Harris KM, et al. Delay from Diagnosis to Surgery in Transferred Type A Aortic Dissection. Am J Med 2018;131:300-6.

9. Harris KM, Strauss CE, Eagle KA, et al. Correlates of delayed recognition and treatment of acute type A aortic dissection: the International Registry of Acute Aortic Dissection (IRAD). Circulation 2011;124:1911-8.

10. Morshuis WJ. Classical Elephant Trunk—old fashioned thus obsolete? J Vis Surg 2021. doi: 10.21037/jovs-20-7.

11. Castrovinci S, Murana G, de Maat GE, et al. The classic elephant trunk technique for staged thoracic and thoracoabdominal aortic repair: long-term results. J Thorac Cardiovasc Surg 2015;149:416-22.

12. Shrestha M, Martens A, Krüger H, et al. Total aortic arch replacement with the elephant trunk technique: single-centre 30-year results. Eur J Cardiothorac Surg 2014;45:289-95; discussion 295-6.

13. Kalra K, Leshnower BG, Chen EP. Narrative review of the thoracic endovascular aortic repairfirst approach for mesenteric malperfusion in type A aortic dissection. J Vis Surg 2022;8:2.

14. Kothari R, Weldon SA, Koksoy C, et al. Narrative review: open surgery for thoracoabdominal aortic aneurysm-is it still a horrible surgery? J Vis Surg 2022;8:4.

15. Coselli JS, LeMaire SA, Preventza O, et al. Outcomes of 3309 thoracoabdominal aortic aneurysm repairs. J Thorac Cardiovasc Surg 2016;151:1323-37.

16. Walker J, Kaushik S, Hoffman M, et al. Long-term durability of multibranched endovascular repair of thoracoabdominal and pararenal aortic aneurysms. J Vasc Surg 2019;69:341-7.

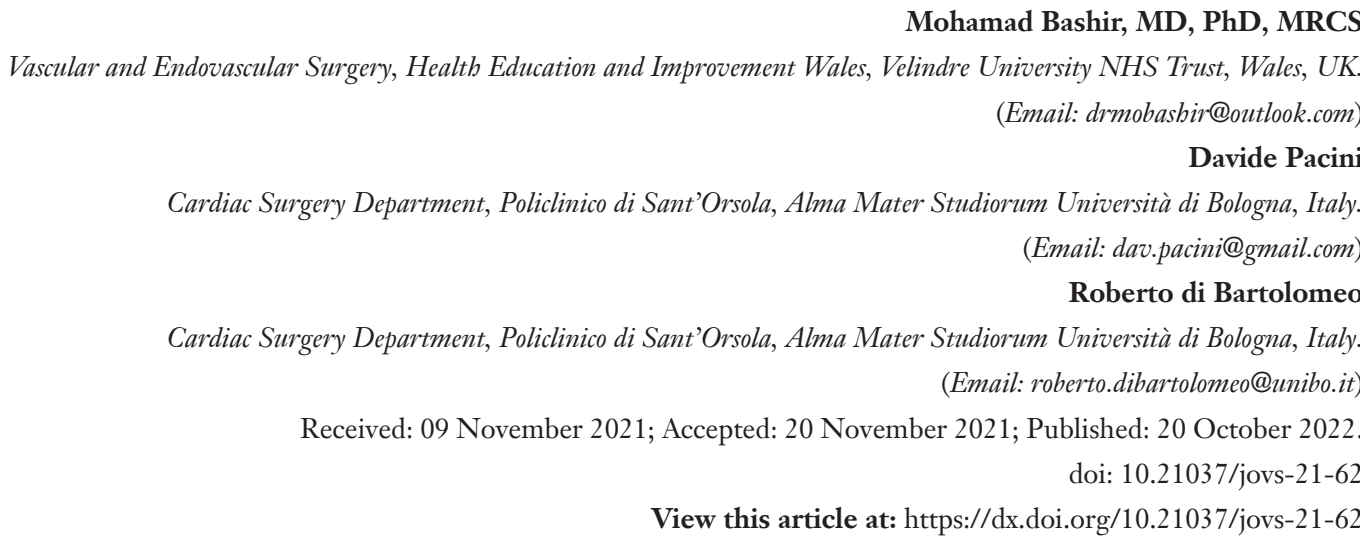

doi: $10.21037 /$ jovs-21-62

Cite this article as: Bashir M, Pacini D, di Bartolomeo R.

Perspectives from Bologna on the future directions of aortic surgery. J Vis Surg 2022;8:32. 